\title{
AN EFFICIENT DEPLOYMENT APPROACH FOR IMPROVED COVERAGE IN WIRELESS SENSOR NETWORKS BASED ON FLOWER POLLINATION ALGORITHM
}

\author{
Faten Hajjej, Ridha Ejbali and Mourad Zaied \\ Research Group on Intelligent Machines (REGIM-Lab) Sfax, Tunisia \\ hajjej.faten.tn@ieee.org \\ ridha_ejbalieieee.org \\ mourad.zaied@ieee.org
}

\begin{abstract}
Wireless Sensor Networks (WSNs) are experiencing a revival of interest and a continuous advancement in various scientific and industrial fields. WSNs offer favorable low cost and readily deployable solutions to perform the monitoring, target tracking, and recognition of physical events. The foremost step required for these types of ad-hoc networks is to deploy all the sensor nodes in their positions carefully to form an efficient network. Such network should satisfy the quality of service $(Q o S)$ requirements in order to achieve high performance levels. In this paper we address the coverage requirement and its relation with WSN nodes placement problems. In fact, we present a new optimization approach based on the Flower Pollination Algorithm (FPA) to find the best placement topologies in terms of coverage maximization. We have compared the performance of the resulting algorithm, called FPACO, with the original practical swarm optimization (PSO) and the genetic algorithm (GA). In all the test instances, FPACO performs better than all other algorithms.
\end{abstract}

\section{KEYWORDS}

WSN, Sensors Deployment problem, Coverage, FPA.

\section{INTRODUCTION}

Technological innovations in miniaturization, power management and wireless communication in the recent years have enabled the progress of wireless networks, which have attracted a growing interest for many applications and fields, such as military sensing, physical air traffic control, video surveillance, traffic surveillance, industrial and manufacturing automation, security, environment monitoring, and building and structural monitoring.

A wireless Sensor Network (WSN), which is a targeted wireless network, consists of a significant number of miniaturized electronic devices, called sensors, distributed over a specified area in order to sense the environment and communicate the accumulated information from the Natarajan Meghanathan et al. (Eds) : NETCOM, NCS, WiMoNe, GRAPH-HOC, SPM, CSEIT - 2016 pp. 117-129, 2016. (C) CS \& IT-CSCP 2016

DOI : $10.5121 /$ csit.2016.61511 
monitored field to other networks (e.g., the internet). In WSN, sensors have limited resources, typically the energy resources, and the calculation capabilities, as well as the storage capacity. Therefore, most studies and researches on WSNs have focused on the optimization of resources in order to enhance the performances and meet the quality of service (QoS) requirements. Determining the sensor field topologies is a key challenge in sensor resource management. Consequently, WSN performance is powerfully influenced by the deployment topology of sensor nodes, which affect QoS metrics, such as energy consumption, sensor lifetime, and sensing coverage equally [1].

In the literature, the deployment topology can be classified according to; the placement methodology that can be either random placement or grid-based placement (deterministic placement), the optimization of performance metrics such as connectivity, sensing coverage, energy consumption and lifetime, and the roles the deployed node, which can be regular, relay, cluster-head, or base-station, plays [2]. However, the placement techniques can be further categorized into static and dynamic whether the optimization is performed at the time of deployment or whiles the network is working, respectively. The choice of the deployment scheme depends on many properties [2]. Therefore, many studies considered that for some cases random placement becomes the only option due to the environment characteristics [3] [4] and deployment cost, and time. Figure 1 shows the different categories of node placement strategies.

Our major focus in this paper is on how to choose the optimal nodes deployment that can achieve maximal coverage of the monitored area [5]. Thus, optimal nodes placement issue is a problem that has been proven NP-hard for most formulations of sensor deployment [6].

The coverage metric is a decisive metric that can be considered as a measure of permanence and QoS for WSN. Coverage in a WSN is to ensure that the Region of Interest (RoI) is monitored with high reliability in order to have the necessary information on the supervised phenomenon [7]. Coverage issues can be commonly classified into two types: target coverage problem and area coverage problem. The former ensures the monitoring of only certain specific points which have fixed positions in the area of interest, while the latter is concerned with the supervision of the whole deployment area. Target coverage can be categorized as Q-coverage or simple coverage. For simple coverage, each target should be monitored by at least one sensor node. For Q-coverage, each target has to be monitored by at least Q different working sensor nodes.

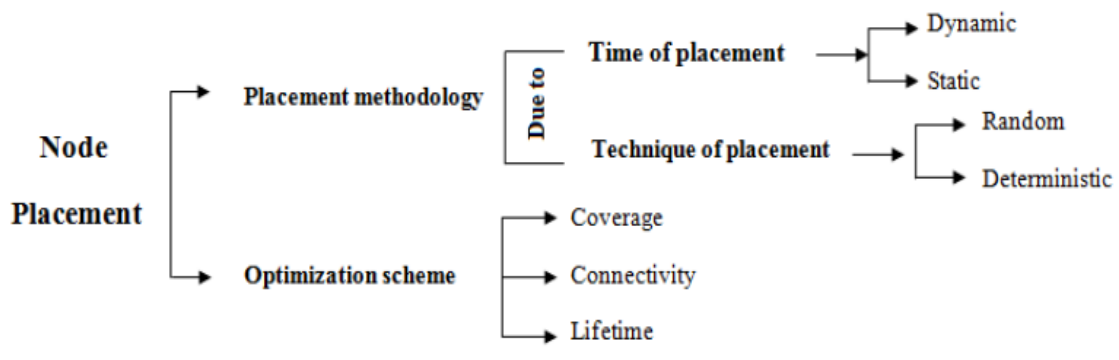

Figure 1. Sensor node placement methodologies

The connectivity metric is as important as coverage in wireless sensor networks. A WSN is defined as connected if, and only if, there exists at least one route between each pair of nodes. Thus, connectivity depends on the existence of paths and can therefore be directly affected by 
changes of topology. For this reason an optimal deployment strategy have to maximize coverage with respect to the connectivity constraint.

Nature constantly inspires research in the field of optimization. While genetics, ants and particle swarm algorithms are famous examples, other nature inspired optimization algorithms emerge regularly. Flower Pollination Algorithm (FPA) is novel global optimization algorithm inspired from pollination process of flowers. FPA is simple and very powerful; in fact, it can outperform both genetic algorithm (GA) and particle swarm optimization (PSO) according to [8].

To find the best nodes deployment that would achieve maximal coverage of the targeted area without affecting network connectivity, a new approach based on FPA is introduced to enhance coverage in a wireless sensor network. We considered a centralized topology and an area coverage problem with random sensor deployment. Here, different scenario was tested. The proposed approach was able to maximize the total coverage area for the considered scenarios. The remainder of this paper is organized as follows. Section 2 gives a literature survey about different deployment algorithms. The problem formulation is presented in section 3. Section 4 specifies the proposed FPA based deployment approach. In section 5, the simulation results and discussion are given. Finally, section 6 concludes the paper.

\section{LITERATURE SURVEY}

Over the last years, researchers attempted to tackle the nodes deployment problem in WSN through various optimization processes both by mathematical programming and through nature inspired techniques. This problem was sometimes modelled as a one single objective problem, in special cases deal with several objectives through well selected weights.

$\mathrm{Yu}$ et al. proposed a node placement algorithm for mobile sensor networks based on the strength of van der Waals in order to improve the total coverage area. In fact, the proximity relationship of nodes is defined by the Delaunay triangulation method, the frictional force is inserted into the equation of force, the force calculated generate an acceleration in the movement of nodes. To evaluate whether the nodes are uniformly distributed over the deployment field an evaluation metric named pair correlation function was introduced in [9]. The Genetic Algorithm (GA) was introduced as a solution for coverage holes problem in WSN [10]. This approach found the optimal positions and the number of mobile nodes that have to be added to the initial deployment schema. Simulation results prove that this algorithm has optimized network coverage in terms of overall coverage ratio and additional number of mobile nodes. Sengupta et al. addressed the problem of achieving an optimal trade-off between coverage, energy consumption, and lifetime in WSN by using the multi-objective evolutionary algorithm (MOEA). They developed an enhanced version of Multi-objective evolutionary algorithm based on differential evolution (MOEA/D-DE) known as MOEA/DFD which includes the fuzzy dominance [11]. Sakamoto et al. proposed a simulation approach founded on Particle Swarm Optimization (PSO). They focused on the size of giant component and number of covered mesh clients (NCMC), which are important objective functions to optimize Wireless Mesh Networks (WMNs) [12]. In their work, the authors of [13] proposed a modified version of the original artificial bee colony $(\mathrm{ABC})$; in fact, they change the updating equation of onlooker bee and scout bee [14]. Indeed, some new parameters, such as forgetting and neighbors factor for accelerating the convergence speed and probability of mutant for maximizing the coverage rate were introduced [15]. Comparing their approach with the deployment topology based on the traditional ABC and PSO algorithm, they found that the 
former achieved better performance in terms of coverage and speed of convergence with less moving distance sensor.

\section{PROBLEM FORMULATION}

The deployment of sensor nodes in WSN is to find the placement nodes topology or find the coordinates of the sensor nodes in the two-dimensional plane. The most important concerns for WSN are how improving the performances and optimizing the resources. Thus, an optimal placement strategy ought to be considered to achieve the required goal. Here our objective is to find an optimal placement schema that maximizes the coverage area without losing network connectivity. For this, the following different mathematical models are described.

\subsection{Preliminary}

Sensor nodes in WSN are characterized by their positions in the 2D plane (x,y), sensing radius $\mathrm{R}_{\mathrm{s}}$, and communication radius $\mathrm{R}_{\mathrm{c}}$. Given a multi-hop WSN, where all nodes collaborate in order to ensure cooperative communication. Such network, can be defined as a linked graph, $G=\{\mathrm{V}$, $E$ \}, where $V$ is the set of vertices representing sensors and $E$ is the set of edges representing links between the sensors. Let $\mathrm{u} \in \mathrm{V}$ and $\mathrm{v} \in \mathrm{V},(\mathrm{u}, \mathrm{v})$ belongs to $\mathrm{E}$ if, and only if, $\mathrm{u}$ can send a message directly to $\mathrm{v}$ (we say that $\mathrm{v}$ is neighbor of $\mathrm{u}$ ). We assume that $\mathrm{R}_{\mathrm{c}}$ is identical for all nodes. Let $\mathrm{d}(\mathrm{u}$, $\mathrm{v})$ be the distance between the nodes $\mathrm{u}$ and $\mathrm{v}$, the set $\mathrm{E}$ can be defined as follows:

$$
E=\left\{(u, v) \in V^{2} ; \mathrm{d}(\mathrm{u}, \mathrm{v}) \leq \mathrm{R}_{C}\right\}
$$

The network coverage is defined by the sensing radius of the sensor nodes, whereas the network connectivity is specified by the communication radius of the nodes.

\subsection{Connectivity}

Definition 1 (Node Degree). Given an undirected graph G. The degree Deg(u), of a vertex $u \in V$ is specified as the number of a vertex $u \in V$ is specified as the number of neighbors of $u$ [16].

Definition 2 (k-Node Connectivity). A graph is considered to be connected if for every pair of nodes, there exists a single hop or a multi-hop path connecting them; otherwise the graph is called disconnected. A graph is considered to be Q-connected if for any pair of nodes there are at least Q reciprocally separate paths connecting them [16].

\subsection{Binary Sensing Model}

The coverage in WSN defined as the total area covered by a set of sensor nodes deployed in the region of interest (ROI). This region is considered as $\mathrm{m} \times \mathrm{n}$ grids, each grid point size was equal to 1 and denoted as $\mathrm{G}(\mathrm{x}, \mathrm{y})$ (Figure. 2). 


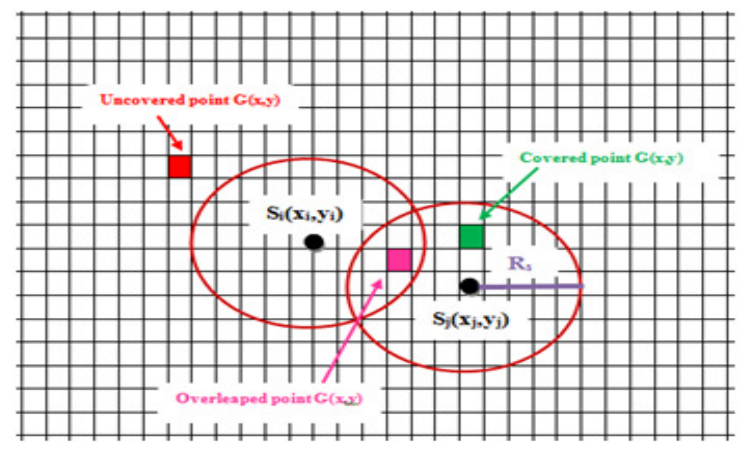

Figure 2. Sensor coverage in sensing field

Generally, the zone covered by a sensor node is a disk with radius equals to sensing radius of the sensor. The binary sensing model considered that each grid point within the sensing radius of a node can be considered as covered with probability equal to " 1 " and the point out of the sensing range was set as "0" since it cannot be covered (Eq1). Thus, the coverage of the whole area is proportional to the grid points that can be covered by at least one sensor $S_{i}\left(x_{i}, y_{i}\right)$ [17].

$$
P= \begin{cases}1, \text { if } & \sqrt{\left(x-x_{i}\right)^{2}-\left(y-y_{i}\right)^{2}} \leq R_{s} \\ 0, & \text { otherwise }\end{cases}
$$

\section{THE PROPOSED APPROACH}

This work interested by the node sensors deployment problem in WSN. In fact, we deal with area coverage problem for random placement topology with predefined number of sensors. Here, the main purpose was to improve the quality of coverage without affecting network connectivity constraint. Evidently, to supply connected coverage to a zone, the set of disks used much cover all points in that region and the connectivity graph of all the $\mathrm{R}_{\mathrm{c}}$-disks must form a single connected component in a graph theoretic sense. The proposed approach, named Flower Pollination Coverage Optimization approach (FPCOA), was a centralized approach based on FPA, aimed to deploy all the sensor nodes in their positions carefully to form a WSN with maximal coverage area.

\subsection{Fitness function}

The binary model was considered as sensing model (Section 2.3). The proposed approach is a mono-objective deployment approach designed to optimize one objective function, namely the ratio of total coverage target area. It is given by:

$$
P(x, y, S)=1-\prod_{i=1}^{N}\left(1-P\left(x, y, S_{i}\right)\right.
$$


With $\mathrm{N}$ is the number of sensor nodes and $\mathrm{P}\left(\mathrm{x}, \mathrm{y}, \mathrm{S}_{\mathrm{i}}\right)$ is the probability that a grid point $\mathrm{G}(\mathrm{x}, \mathrm{y})$ is covered by a sensor $S_{i}$. So, the total coverage area is defined as:

$$
\text { TotCovArea }=\sum_{x=1}^{m} \sum_{y=1}^{n} P(x, y, S)
$$

And the ratio of total coverage area is given by:

$$
\text { Total Coverage ratio }=\frac{\text { TotCovArea }}{\text { TotalGridArea }}
$$

\subsection{Constraints}

The network connectivity is taken as a constraint in this optimization problem. Therefore one path, at least, must exist from the sensor node to the sink node, to guarantee connectivity

\subsection{Flower Pollination Coverage Optimization Algorithm (FPCOA)}

The proposed approach composed of two main steps. The first step was the creation of the initial population (Algorithm 1). The second step was the performing of the optimization process based on FPA (Algorithm 2).

Initial Population. To create the initial population we considered that each individual was represented by a vector of all sensor nodes position (x, y) in RoI. The WSN parameters are described in Table 1.

Table 1. Parameters of WSN.

\begin{tabular}{l|l}
\hline Parameter & Definition \\
\hline $\mathrm{S}_{\mathrm{i}}$ & Node $\mathrm{i}$ \\
$\mathrm{R}_{\mathrm{s}}$ & Node sensing radius \\
$\mathrm{R}_{\mathrm{c}}$ & Node communication radius \\
$\mathrm{x}_{\mathrm{m}}$ & Maximum width of RoI \\
$\mathrm{y}_{\mathrm{m}}$ & Maximum length of RoI \\
$\mathrm{Nsen}_{\mathrm{N}}$ & Number of sensor nodes \\
$\mathrm{NbPp}$ & Number of individual in the initial population \\
$\mathrm{D}_{\mathrm{ij}}$ & Euclidian distance between nodes $\mathrm{i}$ and $\mathrm{j}$ \\
$\mathrm{N}_{\mathrm{e}}$ & Maximum number of neighbors \\
\hline
\end{tabular}


To create initial population, we began by generating the position of the sink node at the centre of RoI (i.e., at $\mathrm{x}_{\mathrm{m}} / 2$ and $\mathrm{y}_{\mathrm{m}} / 2$ ) for each individual. Then, we deployed the remaining sensors by taking into consideration the connectivity constraint. Actually, network connectivity is assumed to be full if the distance between two sensors is less than the communication radius $\left(R_{c}\right)$ of the sensor. The distance is defined as the Euclidean distance between two sensors. In addition, to insure a sufficient distribution in RoI, we controlled the number of neighbors of each deployed node that should be less than a predefined number $\mathrm{N}_{\mathrm{e}}$ (see Algorithm1).

Table 2. Pseudo code of Initial Population Creation.

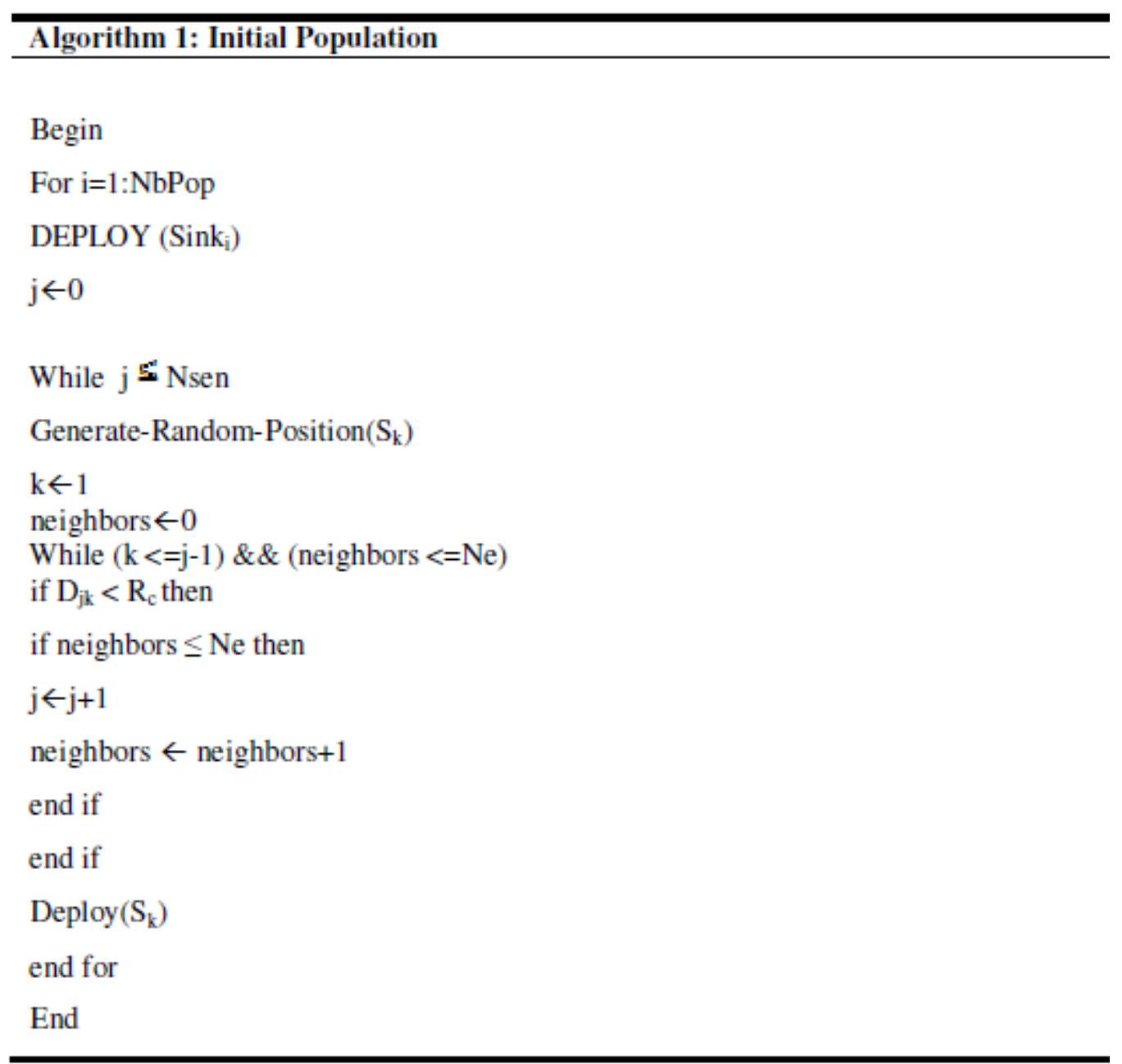

Flower Pollination Algorithm (FPA). metaheuristics are generic algorithms, often inspired from nature, designed to solve challenging optimization problems [17] [18]. Here, we considered one of the most recent metaheuristic algorithms named Flower Pollination Algorithm (FPA), developed by Xin-She Yang in the year 2012 [8] for the global optimization problems. FPA inspired from the flower pollination process of flowering plants. In nature, flowers pollination process resulting from the transfer of pollen, typically, by pollinators such as insects, birds, bats and other animals. In fact, pollination process can be commonly classified into two types: selfpollination and cross-pollination. The former can occur by the pollen of the same flower. The 
latter can take place by pollen of a flower of a different plant [20] [21]. FPA has the following four rules:

1. Cross-pollination is considered as global pollination process with pollen carrying; pollinators performing Lévy flights.

2. Self-pollination is considered as local pollination.

3. Flower constancy can be defined as the reproduction probability is proportional to the similarity of the two flowers involved.

4. Global and local pollination is controlled by a switch probability $p \epsilon[0,1]$.

Table 3. Pseudo Code of Flower Pollination Coverage Optimization.

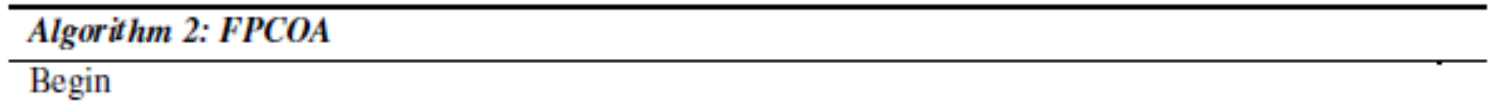

Step 0: Read Nb-flower, switching probability p, MaxIter, solution space

Step 1: create initial population (See Algorithm 1)

Step 2: Perform the optimization process based on FPA

If current iteration $<$ Max-Iter then

for $\mathrm{i}=1$ : Nbflower //all $\mathrm{N}$ flowers in the population

if rand $<$ p then //Global pollination

Draw a (d-dimensional) step vector $\mathrm{L}$ which obeys a Lévy distribution

$f_{\text {Nexti }} \leftarrow f_{\text {Curri }}+L$ (Cunnent-Global-Flower $\left.+f_{\text {Curri }}\right)$

else//local pollination via Randomly choose $j$ and $k$

Draw u from a uniform distribution in $[0,1]$

$\mathrm{f}_{\text {Nexti }} \leftarrow \mathrm{f}_{\text {Cunri }}+\varepsilon\left(\mathrm{f}_{\text {Curnk }}+\mathrm{f}_{\text {Cumj }}\right)$

end if

Total-Coverage-ratio $\left(\mathrm{f}_{\text {Nexti }}\right)$ //Evaluate new solution

If new solutions are better then

update them in the population

Next-Global-Flower $\leftarrow \mathrm{f}_{\text {Nexti }}$

else

Next-Global-Flower $\leftarrow$ Current-Global-Flower

end if

end for

go to Step 2

else Display the set of optimal placement positions

end if

End 
Here each flower was represented by a vector of all sensor nodes position $(\mathrm{x}, \mathrm{y})$ in RoI, $\mathrm{f}_{\text {Curr } 1 \text {, }}$ $\mathrm{f}_{\text {Curr2 }}, \ldots, \mathrm{f}_{\text {Curr }}$ was the flower population at iteration $t, f_{\text {Next1 }}, f_{\text {Next } 2}, \ldots, f_{\text {NextN }}$ was the flower population at iteration $t+1$, Nbflower was the total number of flower and the Current-Global Flower is the best solution found among all solutions at the current generation or iteration t. To imitate the movement of pollinator [22], FPA uses Lévy flight. That is, we draw L $>0$ from a Lévy distribution:

$$
L \sim \frac{\lambda \Gamma(\lambda) \sin \left(\frac{\pi \lambda}{2}\right)}{\pi} \frac{1}{s^{1+\lambda}}\left(\mathrm{s} ? \mathrm{~s}_{0} ? \quad 0\right)
$$

The pseudo-code of FPA is presented in Table3.

\section{SIMULATION AND RESULTS}

To validate the proposed approach, some simulations were undertaken. We used a binary sensing model the nodes are initially randomly distributed. The network is homogeneous, i.e., all sensors have the same deployment parameters such as the sensing and communication radius. Simulations were carried out using MATLAB R2016a. The algorithm was run a maximum number of iterations of 3000. The average of 10 runs was recorded. For the simulations, we considered a square area divided into a number of squares of $1 \mathrm{~m} 2$ each. The center of each of these squares is taken as the demand point to detect by at least one sensor node. In this section, the performance of the proposed FPCOA is evaluated with regard to the total coverage ratio. Moreover, the obtained results were compared with those obtained with two metaheuristics algorithms, namely, PSO and GA and, finally, the effect of the number of randomly deployed sensor nodes was discussed.

\subsection{Efficiency of the proposed approach}

In order to test the performances of FPCOA, we considered a square area with each side $100 \mathrm{~m}$ in length. We considered also that the number of sensors and the communication radius $R_{c}$ as well as the sensing radius $R_{s}$ as constant values. Here the number of sensors was set as $15, R_{c}$ as $15 \mathrm{~m}$ and $R_{\mathrm{s}}$ as $15 \mathrm{~m}$.

Table 4. Deployment results of FPCOA.

\begin{tabular}{l|ccccccc}
\hline $\begin{array}{l}\text { Number of } \\
\text { iterations }\end{array}$ & 0 & 500 & 1000 & 1500 & 2000 & 2500 & 3000 \\
\hline Coverage ratio & 0.491 & 0.649 & 0.891 & 0.926 & 0.951 & 0.968 & 0.973 \\
\hline
\end{tabular}

As seen in Table 4, the effective coverage area was improved significantly over the 3000 iterations. The decrease in the standard deviation values can be explained by the stability of the algorithm with larger numbers of iterations. In fact, FCPOA improved the coverage ratio by 48.2\% compared with the random initial distribution. To highlight this improvement, the best 
deployments obtained by the FCPOA for initial and final configurations are shown in Figure 2 and Figure 3, respectively, where the colored areas represent detected coverage areas.

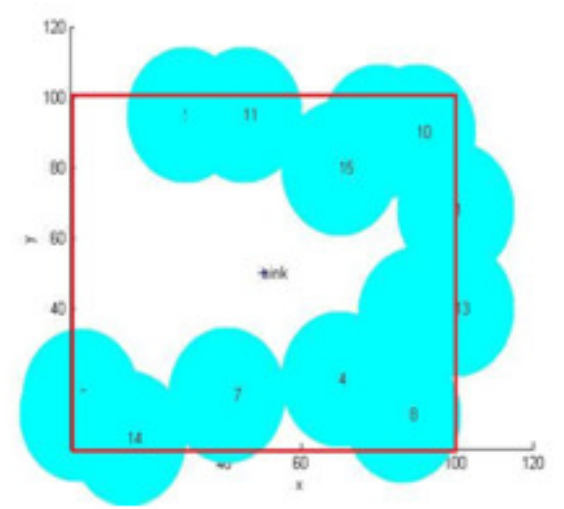

Figure 3. Initial configuration of Sensors

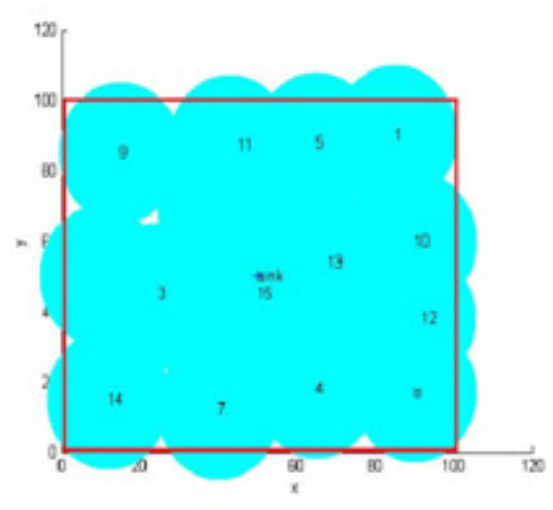

Figure 4. Final configuration of Sensors

\subsection{Comparison with other approaches}

To evaluate the efficiency of our proposed approach we choose to compare our results with those obtained with GA and PSO, respectively. Figure 5 gives the comparison of the coverage rate tested on the same initial population for the three approaches.

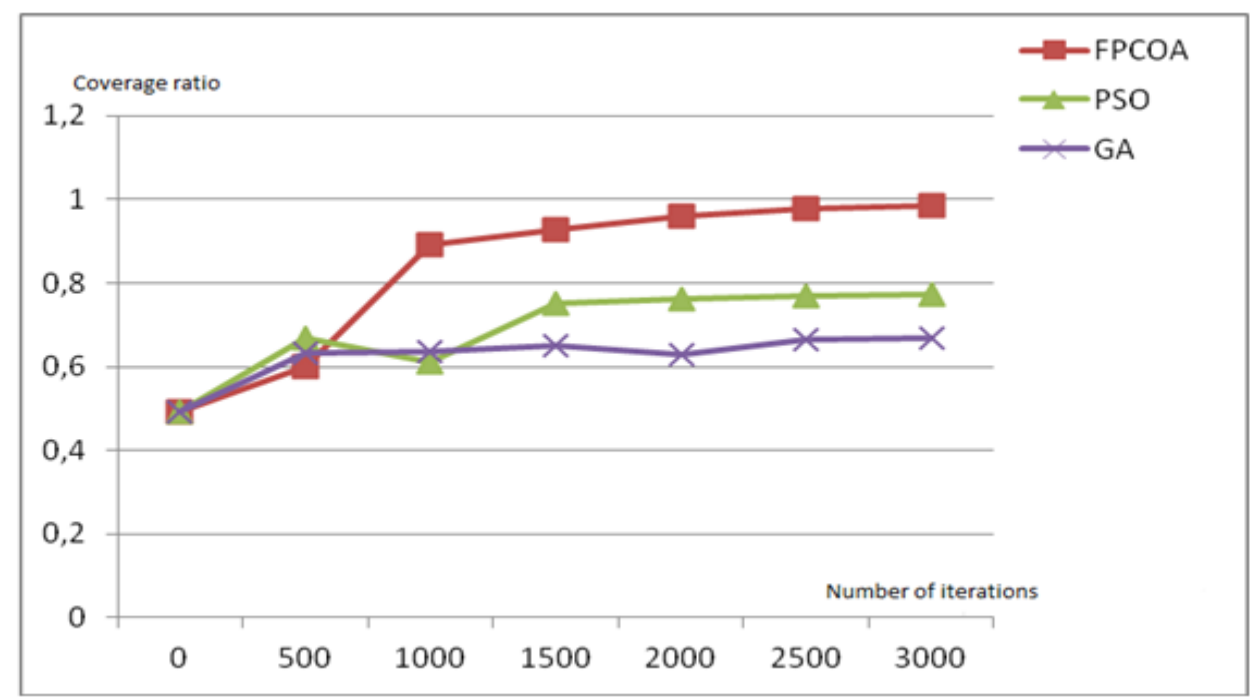

Figure 3. Comparison of total coverage ratio with GA and PSO

From this figure, we can find that after the nodes reached stable distribution and obtained the optimal placement topology, the proposed algorithm has better coverage rate than the other two approaches. The results of the proposed approach clearly outperform both than GA and PSO respectively. This figure shows that FPCOA gives a much more stable performance in total coverage than both the two algorithms. 


\subsection{Effect of Number of Sensor Nodes}

In order to show the effect of number of sensor on the total coverage ratio for the propose approach, we considered that the sensor nodes were randomly deployed in a $50 \mathrm{~m} \times 50 \mathrm{~m}$ sensor field, the communication radius $R_{c}$ was set as $5 \mathrm{~m}$ and the sensing radius $R_{s}$ was set as $5 \mathrm{~m}$.

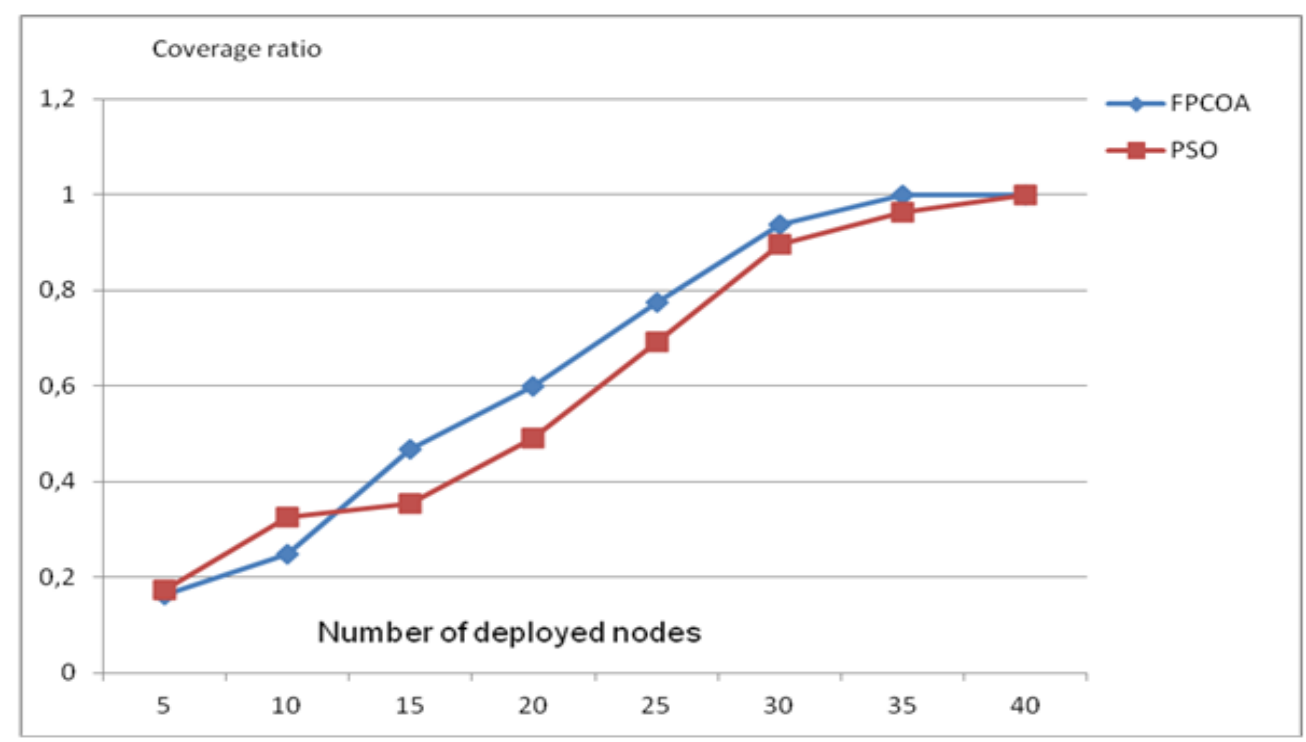

Figure 4. The Coverage Ratio vs. Number of Sensor Nodes

Figure 6 shows the coverage ratio when adding sensor nodes to the network for both of FPCOA and PSO. As shown, the coverage ratio increases as the number of deployed nodes increases. This figure indicates that the proposed approach offers higher coverage with less sensor nodes. FPCOA requires around 32 sensor nodes to get 100\% coverage compared to PSO which requires 34 sensor nodes. Thus, it can be said that FPCOA is able to offer higher coverage with the lowest cost.

\section{CONCLUSION}

In this paper, the sensor placement problem for WSN is addressed. A deployment approach based on FPA was proposed. This approach can find the optimal placement topology in terms one QoS metric. The simulations results of the different scenarios prove that our proposed approach achieved the optimal placement regarding coverage maximization and connectivity constraint. In a future work, we will incorporate other QoS metrics like energy consumption and deal with multi-objective node placement problem for the WSNs.

\section{REFERENCES}

[1] F. Oldewurtel, P. Mhnen, (2010) "Analysis of enhanced deployment models for sensor networks," in Vehicular Technology Conference, pp. 1-5. 
[2] M. Younisa, K. Akkayab. (2008) "Strategies and techniques for node placement in wireless sensor networks: A survey”, Ad Hoc Networks. Vol. 6, No. 4, pp. 621-655.

[3] F. Y. S. Lin, P. L. Chiu, (2005) "A near-optimal sensor placement algorithm to achieve complete coverage-discrimination in sensor networks", IEEE Communications Letters, Vol. 9, No. 1, pp. 4345.

[4] M. Zaied, C. Ben Amar, M. A Alimi "Award a new wavelet based beta function" International conference on signal, system and design, SSD03 1, 2003, pp. 185-191 .

[5] R Ejbali, Y Benayed, M Zaied, A. M Alimi "Wavelet networks for phonemes recognition" International conference on systems and information processing , 2009.

[6] L. W. X. Cheng, D-Z Du, B. Xu, (2008), "Relay sensor placement in wireless sensor networks", Journal of Wireless Networks, Vol. 14, No. 3. pp. 347-355.

[7] R. G. J. Wang, S. Das, (2010) "A survey on sensor localization". Journal of Control Theory and Applications, Vol. 8, No.1, pp.2-11.

[8] X. S. Yang, "Flower pollination algorithm for global optimization" in Unconventional Computation and Natural Computation, vol. 7445, 2012, pp.240-249.

[9] X. Yu, N. Liu, W. Huang, X. Qian, and T. Zhang, (2013) "A node deployment algorithm based on van der waals force in wireless sensor networks", International Journal of Distributed Sensor Networks, pp. 1-8.

[10] O. Banimelhem, M. Mowafi, W. Aljoby, (2013) "Genetic algorithm based deployment in hybrid wireless sensor networks", Communications and Network, Vol. 5 No. 4, pp. 273-279.

[11] S. Senguptaa, S. Dasb, M.D. Nasira, B.K. Panigrahic, (2013) "Multi-objective node deployment in wsns : In search of an optimal trade of among coverage, lifetime, energy consumption, and connectivity", Engineering Applications of Articial Intelligence, Vol. 26, No. 1, pp. 405-416.

[12] S. Sakamoto, T. Oda, M. Ikeda, L. Barolli , (2015) "Design and implementation of a simulation system based on particle swarm optimization for node placement problem in wireless mesh networks," Intelligent Networking and Collaborative Systems (INCOS), pp. 164-166.

[13] X. Yu, J. Zhang, J. Fan, and T. Zhang, (2013) A faster convergence artificial bee colony algorithm in sensor deployment for wireless sensor networks, International Journal of Distributed Sensor Networks, Vol. 9, No. 10 pp. 2-9.

[14] B. Guedri, M. Zaied, C. Ben Amar "Indexing and images retrieval by content" High Performance Computing and Simulation (HPCS), pp. 2011.

[15] A. El Adel, M. Zaied, C. Ben Amar "Learning wavelet networks based on Multiresolution analysis: Application to images copy detection Communications", Computing and Control Applications (CCCA), 2011.

[16] D. B. West, (2006) "Introduction to graph theory", 2nd ed., Prentice-Hall.

[17] A. Hossain, P. K. Biswas, S. Chakrabarti, (2008) "Sensing Models and Its Impact on Network Coverage in Wireless Sensor Network," pp1-2. 
[18] M. Zaied, R. Mohamed, C. Ben Amar, (2012) "A power tool for Content-based image retrieval using multiresolution wavelet network modeling and Dynamic histograms", International REview on Computers and Software (IRECOS), vol. 7 No. 4.

[19] R. Ejbali, M. Zaied, C. Ben Amar, (2012) "Multi-input Multi-output Beta Wavelet Network: Modeling of Acoustic Units for Speech Recognition", International Journal of Advanced Computer Science and Applications (IJACSA), Vol. 3, No. 4.

[20] A. Elfes, "Occupancy grids: a stochastic spatial representation for active robot perception," in Autonomous Mobile Robots: Perception, Mapping and Navigation, vol. 1, S. S. Iyenger and A. Elfes, Editors, IEEE Computer Society Press, pp. 60-70, 1991.

[21] O. Jemai, R. Ejbali, M. Zaied, C. Ben Amar "A speech recognition system based on hybrid wavelet network including a fuzzy decision support system" Seventh International Conference on Machine Vision (ICMV), pp. 503-944, 2015.

[22] R. Ejbali, M. Zaied, C. Ben Amar, "Intelligent approach to train wavelet networks for Recognition System of Arabic Words", International Conference on Knowledge Discovery and Information Retrieval, 2010.

\section{AUTHORS}

Faten Hajjej received the graduate degree in Computer Engineering from the National School of Engineers of Sfax, University of Sfax, in 2009. She has been pursuing the Ph.D degree with the Research Group on Intelligent Machines (REGIMLab), University of Sfax, under the supervision of Dr. Ridha EJBALI and Prof. Mourad Zaied. His research interests include the internet of things, wireless sensor network, multi objective optimization, nature inspired optimization algorithms.

Ridha Ejbali received the Ph.D degree in Computer Engineering, Master degree and computer engineer degree from the National Engineering School of Sfax Tunisia (ENIS) respectively in 2012, 2006 and 2004. He was assistant technologist at the Higher Institute of Technological Studies, Kebili Tunisia since 2005. He joined the faculty of sciences of Gabes Tunisia (FSG) where he is an assistant in the Department computer sciences since 2012. His research area is now in pattern recognition and machine learning using Wavelets and Wavelet networks theories.

Mourad Zaied received the HDR, the Ph.D degrees in Computer Engineering and the Master of science from the National Engineering School of Sfax respectively in 2013, 2008 and in 2003. He obtained the degree of Computer Engineer from the National Engineering School of Monastir in 1995. Since 1997 he served in several institutes and faculties in university of Gabes as teaching assistant. He joined in 2007 the National Engineering School of Gabes (ENIG) as where he is currently an associate professor in the Department of Electrical Engineering. He is a member of the REsearch Group on Intelligent Machines laboratory (REGIM) http://www.regim.org in the National
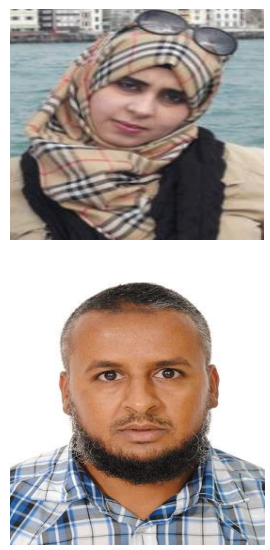
Engineering School of Sfax (ENIS) since 2001. His research interests include

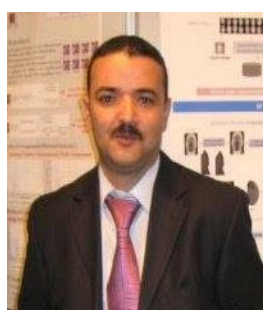
Computer Vision and Image and video analysis. These research activities are centered on Wavelets and Wavelet networks and their applications to data classification and approximation, pattern recognition and image, audio and video coding and indexing. 